Alternate-day prednisone reduces morbidity and improves pulmonary function in cystic fibrosis. Lancet 1985;ii:686-8.

P Caiger, G J Frost, L E Bruce, and S G F Wilson Departments of Biochemical Medicine and Child Health, Ninewells Hospital, Dundee DDI $9 S Y$

\section{Spermatic cord torsion}

Sir,

In his recent annotation Matthews raises a number of points that require correction. ${ }^{1}$ The statement that neonatal spermatic cord torsion occurs "especially in the premature baby' is not borne out by any other authors on the topic. and, in fact, in 62 patients in the published reports for whom information is available the mean birth weight was $3600 \mathrm{~g}$. In fact, this condition seems to be confined to term babies and, indeed, in general to large term babies. While I agree that surgery to fix the contralateral testis is mandatory in these infants, urgent surgery to attempt detorsion of the twisted testis is not necessary in most instances as the testis is almost invariably beyond salvage. In 25 instances in the published reports where detorsion and retention of the testis was performed there were only two occasions where testicular survival was thought to have occurred. (Burge DM. Neonatal testicular torsion and infarction: aetiology and management. Unpublished data.)

The comment that idiopathic scrotal oedema is a rare condition is slightly misleading. Of the children referred to this unit from the Southampton district in the years 1980-85, there were 46 children with testicular torsion, 22 children with torsion of hydatid of Morgagni, and 31 with idiopathic scrotal oedema. Other authors have noted that this condition is probably the second commonest cause of acute scrotal disease that leads to admission to hospital. ${ }^{2}$ Considerable experience in making this diagnosis is required, however, before conservative management is adopted.

I wholeheartedly agree with the comments made regarding epididymo-orchitis and the worrying frequency with which this extremely rare condition in the paediatric age group is still being diagnosed by referring practitioners. The medical school teaching that all acute scrotal disease requires surgical exploration is still by far the safest starting point in the management of a child with scrotal pain.

\section{References \\ 1 Matthews PN. Spermatic cord torsion. Arch Dis Child 1986;61:426-7. \\ 2 Brereton RJ, Manley S. Acute scrotal pathology in boys. Z Kinderchir 1980;29:343-57.}

D M BURGe Southampton General Hospital, Southampton SO9 $4 X Y$

Dr Matthews comments:

Thank you for correctly pointing out that neonatal spermatic cord torsion usually occurs in the full term baby.
I still believe that urgent surgery offers the best chance of testicular salvage and although I concede that more babies will be lost than saved, there is nothing to be gained by delay, and with more widespread adoption of an urgent surgery policy maybe we could improve on the rather dismal testicular salvage rates in the neonate.

I agree that idiopathic scrotal oedema is more common that was originally thought but felt that non-diagnosis of this strange condition was preferable to wrongly diagnosed testicular torsion.

\section{Cost of Stycar boxes}

Sir,

I was extremely surprised to find that the Stycar Vision and Hearing test equipment cost $£ 95.00$ and $£ 59.95$, respectively. These boxes consist of a few toys, rattles, polystyrene balls, and standard cards for vision and hearing checks.

The rattles break often and the toys go missing frequently, and I am sure it is a very profitable business for the manufacturers.

I hope all of us who use Stycar boxes realise their cost and ask ourselves and the manufacturers why they cost so much.

A Thenabadu New Malden. Surrey

\section{Mr M Jackson comments:}

We are grateful to Dr Thenabadu in raising this question but are rather upset at the suggestion that Stycar offers only 'a few toys, rattles, etc'.

The Stycar series of tests, Hearing, Vision and Language, have, of course, proved of immense value to practitioners over the years in helping them to obtain reliable information about children's capacity in hearing comprehension and visual comprehension and acuity and their ability to comprehend and express themselves in the spoken language.

The components and style of packaging have been those that suited the needs of practitioners but it is perhaps important that over the last 12 months or so a slightly different pattern of requests has begun to emerge and we have recently been in touch with many paediatricians and medical officers to identify if a repackaging is required. The current 'make up' of the Stycar tests and style of packaging is certainly not cheap-partly because several of the items need to be especially prepared and in such small quantities that high costs are inevitable.

If we are able to identify accurately alternative and more economic means of presentation then we will be only too happy to examine them because, ironically enough and in direct contradiction to what Dr Thenabadu suggests, the present format of these tests is most certaintly not profitable to us.

NFER-Nelson do operate responsibly and we fully appreciate that there are probably many 'costs' and other 
factors that may not be obvious or apparent to our customers. We would have been happy to talk these through with $\mathrm{Dr}$ Thenabadu if she had contacted us and would certainly be pleased to hear how she uses the test items in day to day practice. It is important also to mention that NFER-Nelson are currently undertaking a major new product aimed at providing practitioners with a totally new developmental screening procedure for the 0-5 year olds based on the Stycar Sequences. It has been in the course of our widespread discussion of this that so much useful new information has come back to us concerning the current usage of the existing Stycar tests.

\section{Vision testing beads}

Sir,

I wish to report a simple device for screening the visual acuity of children over 6 months old. The device itself is constructed from four spherical white beads (approximately $2 \mathrm{~mm}, 3 \mathrm{~mm}, 6 \mathrm{~mm}$, and $12 \mathrm{~mm}$ in diameter) that are strung between two small spools on a $70 \mathrm{~cm}$ length of dark 3/0 monofilament thread (Figure). When not in use, the device can be stored in a small container, with the thread being wound onto one or other spool to prevent tangling.

While sitting facing the child, the clinician palms one spool in each hand, stretching the thread taut and roughly horizontal just in front of a dark background (such as an upright black attaché case on the clinician's lap). All but one bead is trapped against the spools, and the remaining bead is shuttled back and forth by raising first one and then the other hand. The child's visual acuity can be assessed from how small a bead elicits visual tracking when the beads are 3 metres (or 1.5 metres) away.

For children aged 18 months or over, the clinician's clothing or case can provide a suitable dark background. Children under the age of 18 months generally find the clinician's face more interesting than the shuttling bead. This problem can be overcome by using a small screen with an eye slit mounted on the lap.

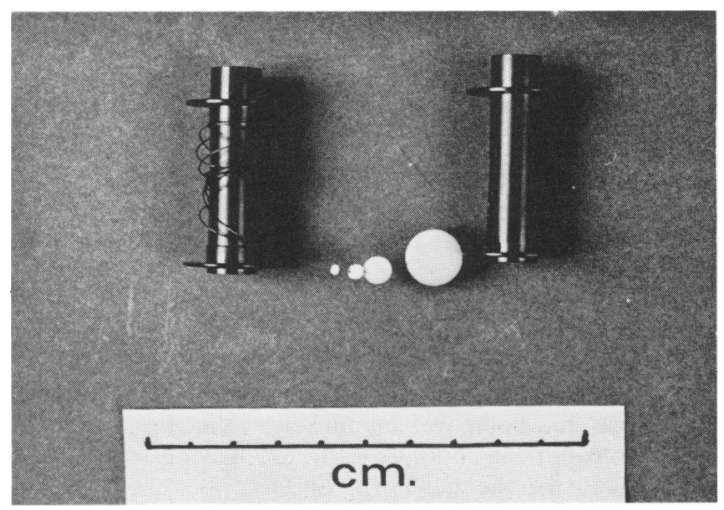

Figure Close up of vision testing beads.

Preliminary field trials indicate that Sheridan's norms ${ }^{1}$ for the Stycar graded balls can be transferred to sliding beads: the normal 6 month infant can track a $6 \mathrm{~mm}$ bead at 3 metres (or a $3 \mathrm{~mm}$ bead at 1.5 metres), and from 8 months of age upwards normal children can track a $3 \mathrm{~mm}$ bead (and often a $2 \mathrm{~mm}$ bead) at 3 metres.

The vision testing beads are cheap, compact, and easy to make and use. They have some advantages over the standard Stycar techniques: no need for a room large enough to accommodate a long black mat (unlike the Stycar 'rolling balls' technique) and no need for a large screen and a room with one black wall (unlike the Stycar 'mounted balls technique').

\section{Reference}

' Sheridan MD. The Stycar graded-balls vision test. Dev Med Child Neurol 1973;15:423-32.

R Goodman Maudsley Hospital, London SE5 8AZ 